\title{
A Scheme of sEMG Signal Channel Reduction for Upper Limb Motion Recognition
}

\author{
Yanzhao Chen and Yu-Wei Zhang \\ School of Mechanical and Automotive Engineering, Qilu University of \\ Technology, Jinan, China \\ E-mail: chyzh_ql@126.com
}

\begin{abstract}
The accuracy and real-time of motion classification are two essential aspects of sEMG signal based motion recognition, which is widely used in robot-assistant upper limb rehabilitation. The number of channels used for signal acquisition is a key factor affecting the real-time of motion classification. The selection of channels in current researches is usually based on experience or experiments without a consistent theoretical foundation. In order to enhance the real-time of motion recognition, the channel reduction scheme is constructed in this paper. After evaluation of the channel contribution to motion classification by an improved ReliefF algorithm and CSI index, the channel reduction principles are proposed. The experimental result shows that the motion classification time has reduced significantly without adversely affecting the classification accuracy, which also simplifies the signal acquisition process and facilitates the development of the following clinical rehabilitation products. The result proved the proposed channel reduction method is effective and significant.
\end{abstract}

Keywords: sEMG; signal channel reduction; upper limb; pattern recognition; ReliefF

\section{Introduction}

Stroke and other cerebrovascular diseases often cause paralysis, especially that the patients' upper limbs cannot move freely, which affect their activities of daily living [1, 2]. Carrying out rehabilitation training will help relieve their disease as well as reduce the burden of their families and the society. Medical research shows that repetitive movements training can help patients recover their voluntary movement function, which become a generally adopted means of rehabilitation in clinic [3,4]. With the development of computer science and biomedical engineering, robot-assisted upper limb selfrehabilitation training can overcome the shortcomings of traditional training methods, which causes wide concerns [5-7]. The motor function of one side of the patient's upper limb is generally blocked. Leading the disabled side to execute motion training under the assistance of a rehabilitation robot, which is guided by the healthy side of upper limb, is a viable way of rehabilitation. With the development of biomedical technology, various physiological signals are applied in rehabilitation medicine, among that the sEMG signal $[8,9]$ is more suitable for medical applications as it is rich in the information of human's muscle status, which can reflect human's neuromuscular activity and movement intent. In recent years, sEMG is commonly used in the field of rehabilitation medicine [10, 11].

sEMG signal based pattern recognition is the key technique to achieve robot assisted upper limb rehabilitation training. The general procedure is: firstly, acquire the sEMG signal from the healthy upper limb when executing movement; secondly, identify the motion type from the signal by pattern recognition and then convert that result to the robot control instructions; finally, achieve the robot-assisted upper limb exercise training.

Received (May 23, 2016), Review Result (November 19, 2016), Accepted (December 6, 2016) 
Acquiring the sEMG signal by multiple channels from different muscle positions becomes a widely used manner for pattern recognition. Such as American scholars Alan Smith and Edward E. Brown [12] reported a control program for a robot with three degrees of freedom. They acquired the signals from biceps, triceps and four muscles on the forearm by multiple channels and identified the motions. After feature extraction and classifier design, the motion classification experiment was executed, and the classification accuracy reaches more than $90 \%$. Australian scholars Rami N. Khushaba, etc. [11] accurately identified the finger movement by sEMG signal to drive a prosthetic hand performing different movements. The researchers acquired sEMG signal from the forearms of eight subjects in two channels, the motion recognition accuracy reached more than $90 \%$. Although acquiring sEMG signal from multiple channels may get higher recognition accuracy, there will be some problems with an excessive number of channels. Firstly, during the sEMG signal acquisition, because of the surface area limitation of the upper limb skin, it will inevitably cause crosstalk among channels, which will generate invalid information. Secondly, when the upper limb executes movement, one muscle involved in multiple motions has different characteristics; meanwhile, one motion is generated by many muscles' interaction, some of which have similar functions and exhibit similar characteristics $[13,14]$. In other words, the functions to characterize one motion patterns of signals from different channels may be the same or similar. Although this phenomenon increases the feasibility of motion identification, it will produce redundancy among signals from different channels. These redundant signals will show low contribution in the subsequent pattern classification, which will not achieve promotion on classifications or even be counterproductive. Reducing the number of signal channels can decrease the time complexity of pattern classification, which will enhance the real-time of classification. In addition, reducing the number of signal channels will bring convenience to sEMG signal acquisition, which also has important significance for the development of portable clinical products.

Previous studies have shown that the signal acquisition position has important influence on classification results, and using a small amount of electrodes to obtain high classification accuracy is very useful for clinical myoelectric control [13, 15-16]. However, these studies mostly rely on experience or experiments to select the signal channel and signal acquisition positions, which do not point out a united principles and basis. ReliefF [17] is a multivariable selection algorithm, which is usually used for feature selection. Meanwhile, the sEMG signal channel reduction can be seen as an issue of combination of feature selection. In this article, an improved ReliefF algorithm is provided for implementing sEMG signal channel reduction.

\section{Materials and Methods}

The process of sEMG based upper limb motion recognition can be divided into the following steps: signal acquisition, feature extraction and motion classification. In our preliminary studies [18], six upper limb motion types were chosen for rehabilitation training, which are elbow flexion, elbow extension, shoulder flexion, shoulder extension, shoulder abduction as well as shoulder adduction. The sEMG signals were acquired in six channels from the upper limb, which are central deltoid, rear deltoid, biceps, triceps, brachioadialis and flexor carpi. The sEMG signal acquisition instrument ME6000 made in Finland was used for signal acquisition with six channels. Each signal acquisition channel consists of three electrode interfaces, two of which are used to connect the measuring electrodes and the other to connect the reference electrodes to form a bipolar differential setting. During the signal acquisition, the three electrodes of each channel were pasted to the skin surface near the corresponding muscle. A feature space BisIE based on bispectrum technique is constructed, and the SVM was chosen as pattern classifier. In this chapter, the scheme of channel reduction is proposed, based on which the number of 
channel will be reduced. The algorithm implementation in this paper is based on MATLAB2012a and windows operating system. The authors declare that all experimental procedures executed in this research comply with the requirements of the local ethics committee and the declaration of Helsinki.

\subsection{Channel Reduction Scheme}

In the feature space, the classification contributions of features from different channels may be different, based on which the signal channel reduction scheme is proposed in this section. The classification contribution is taken as standard. This scheme is shown in Figure 1. The channel reduction process can be described as: firstly, make the feature extraction from each channel and, after feature extraction, there will be the feature space of six channels. For BisIE, the feature space is 18-dimensional with 3 dimensions each channel; secondly, calculate the contribution of each channel for motion classification and sort the channels in descending order according to the degree of contribution; thirdly, get the combinations of different channels based on reverse culling method and achieve channel selection by analyzing the feature separability; finally, determine the channel combination after reduction.

When determining the final channel combination, the following principles should be considered:

(1) The positions of muscles that correspond to different signal channels should be as far as possible, in order to facilitate the signal acquisition and avoid interference.

(2) For the antagonistic muscles, the strength changes of them will be in opposite or relaxed state when executing reverse motions. Their contributions to motion classification may be the same or similar, which makes it possible to retain one of them as the signal acquisition position.

(3) For the signals acquired from various types of motions execution, only one channel needs to be retained if large correlation occurs among them, in order to reduce the redundancy.

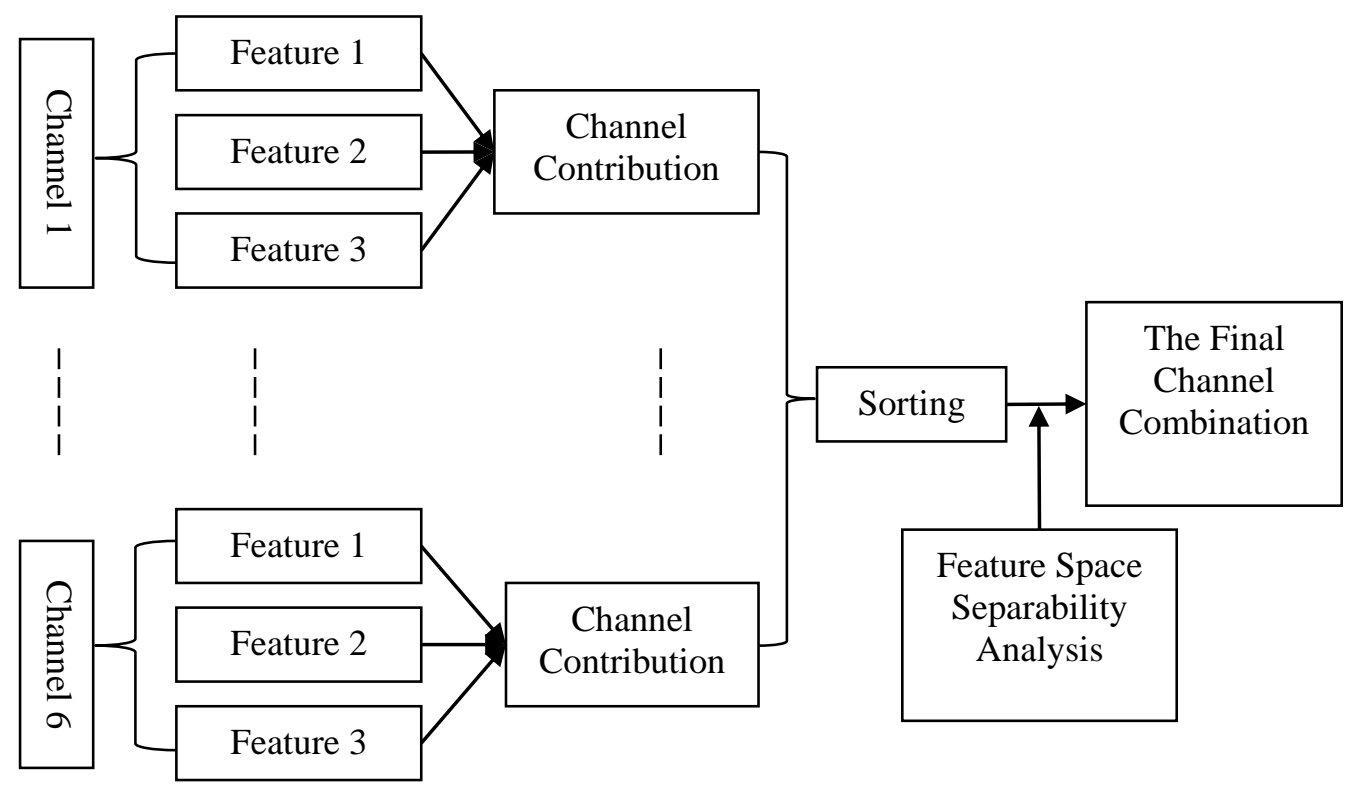

Figure 1. The Scheme of Channel Reduction 


\subsection{The Introduction of ReliefF}

The channel selection can be seen as an issue of special combined feature selection. First proposed by Kira and Rendell [17] in 1992, Relief is a multivariate feature selection algorithm based on sample feature weight. The core of this algorithm is to determine the feature weight based on the difference between the same type of sample and the different types of sample, which reflects the degree of association by the features' ability to distinguish the similar samples. If the difference of the feature is small in the same type of sample and large in different types of sample, the separability of this feature is considered to be strong and vice versa.

Designed for two-class issue, the Relief algorithm can't be used directly in the upper limb classification, which is a muti-class issue. The ReliefF algorithm proposed by Kononenko [19], solved the multi-class issue by transforming it into an one-against-rest issue. The ReliefF algorithm can be described as follows:

Set $X=\left\{x_{1}, x_{2}, \cdots, x_{n}\right\}$ as a sample collection, and each sample contains $\mathrm{m}$ features, that is $x_{i}=\left\{f_{1}, f_{2}, \cdots, f_{m}\right\}$, with $C=\left\{c_{1}, c_{2}, \cdots c_{l}\right\}$ is the class collection with 1 classes that the sample belongs to.

(1) For each feature variable, initialize the weight $w_{j}=0 \quad(1 \leq j \leq m)$;

(2) Randomly select a sample from $x_{i}(1 \leq i \leq n)$, for the feature variable $f_{j}$

$1 \leq j \leq m$ ) of $x_{i}$, select d samples closest to ${ }^{x_{i}}$ from the same type of sample and constitute the sample collection $\mathrm{H}$. Then, select d samples closest to ${ }^{x_{i}}$ from the different types of sample and constitute the sample collection $\mathrm{M}(\mathrm{c})$;

(3) Update the weight of feature variable according to formula (1):

$$
\begin{aligned}
w_{i}= & w_{i}-\sum_{n=1}^{l} \operatorname{diff}\left(f_{j}, x_{i}, H_{n}\right) /(t \times d)+ \\
& \sum_{c \notin \text { class }\left(x_{i}\right)}\left[\frac{p(c)}{1-p\left(\operatorname{class}\left(x_{i}\right)\right)} \sum_{n=1}^{l} \operatorname{diff}\left(f_{j}, x_{i}, M_{n}(c)\right)\right] /(t \times d)
\end{aligned}
$$

(4) Turn to (2) and iterate $2 t(1<t)$ times;

(5) Calculate the feature weights of all feature variables to obtain the weight vector $\mathrm{W}$;

(6) Sort the elements of the weight vector in descending order and select the features with weights greater than a threshold value $\mathrm{C}$ as the target features.

\subsection{ReliefF based sEMG Signal Channel Effectiveness Determination}

Improve the ReliefF algorithm and consider the signal channels as a unit, and then, calculate the feature weights of different channels, the results of which were seen as the contribution degree for the motion classification. For BisIE feature space, there are 6 channels with 3 features in each channel, resulting in 18 features totally. The process of the weight calculation of each channel by improved ReliefF algorithm is as follows:

(1) Respectively select each feature in each channel composing the input vectors $F_{i}=\left[f_{1}, f_{2}, \cdots, f_{6}\right]$, where i represents the feature number, and $1 \leq i \leq 3, f_{j}$ represents the $\mathrm{i}$-th feature of the $\mathrm{j}$-th channel;

(2) Calculate the feature weights $W_{i}=\left[w_{1}, w_{2}, \cdots w_{6}\right]$ of each channel, $w_{j}$ represents the $\mathrm{i}$-th feature weight of the $\mathrm{j}$-th channel, $1 \leq j \leq 6$;

(3) Calculate the mean value of feature weights of each channel according to formula (2) as the weight of the corresponding channel, which is the basis of channel reduction. 


$$
W=\frac{1}{3} \sum_{i=1}^{3} W_{i}
$$

\subsection{Feature Separability Analysis}

Through the ReliefF algorithm, the weights of channels are obtained. It is generally considered that the channel with a large weight will have strong discriminatory ability as well as high effectiveness for sample classification and vice versa. After the channel effectiveness sorting according to channel weight, the classification capability of feature space of different channel combinations should be evaluated. Theoretically, the feature space separability can be used to evaluate the discriminatory ability of feature space. Clustering Separation Index (CSI) is a generally used feature separability index, which is also employed in this study..

The method for calculating the feature space separability by CSI is as follows:

Firstly, calculate the within-class scatter $S_{i i}$, which is used to measure the degree of dispersion between motion samples of the same type, and the between-class scatter $S_{i j}$, which used to measure the mean distance of feature vector between two types of motion by:

$$
\begin{gathered}
S_{i i}=\sqrt{\frac{1}{N_{i}} \sum_{k=1}^{N_{i}}\left\|X_{k}-M_{i}\right\|^{2}} \\
S_{i j}=\left\|M_{i}-M_{j}\right\|
\end{gathered}
$$

Where, $X_{k}$ refers to the samples in class $i, N_{i}$ refers to the number of samples in class $i$, and $M_{i}$ refers to the mean value of feature vector of the $\mathrm{i}$-th class feature vector.

Secondly, calculate the similarity $R_{i j}$ between two types of sample. The smaller the $R_{i j}$ is, the easier it will distinguish the two types of sample.

$$
R_{i j}=\frac{S_{i i}+S_{j j}}{S_{i j}}
$$

Finally, determine the overall separability feature space by CSI. The CSI index set the mean value of between-class scatter between two motion types most difficult to distinguished as the sample separability, and the smaller its value is. The smaller its value is, the better its separability:

$$
C S I=\frac{1}{K} \sum_{i=1}^{K} \max _{i \neq j} R_{i j}
$$

To determine the final channel combination, the separability of different channel combinations is examined. Excluding the last channel in the channel sequence successively, the CSI value of the remaining channels is calculated as a combination, resulting in CSI values of six combinations containing one channel to six channels respectively. Based on the separability analysis by CSI value and considering other related factors, the channel reduction can be finished.

\section{Results}

Five healthy subjects took part in the experiment and six upper limb motions were executed by them respectively. Each subject executes every motion for 60 times in three groups with 20 times each, creating 360 samples for each subject of six motions. The 
sEMG signals were acquired in six channels preliminarily and the BisIE feature was extracted. The samples were equally divided into two parts: one was used for training, and the other was used for testing. The channel reduction method proposed above was adopted and the number of channels was reduced.

\subsection{The Channel Contribution}

The weights of channels are calculated by the improved ReliefF algorithm. In order to reflect the distribution and trend of feature weights among different channels clearly, the percentage of weight for each one in all channels was calculated by formula (7) as the channel efficiency rate.

$$
w^{j}=\frac{W^{j}}{\sum_{k=1}^{6} W^{k}}
$$

Where, $W^{j}$ refers to the weight of the j-th channel.

The channel efficiency rate can be seen as channel contribution. As shown in Figure2, the weight rate distributions among different channels of different subjects have a similar trend, although they are not exactly the same.

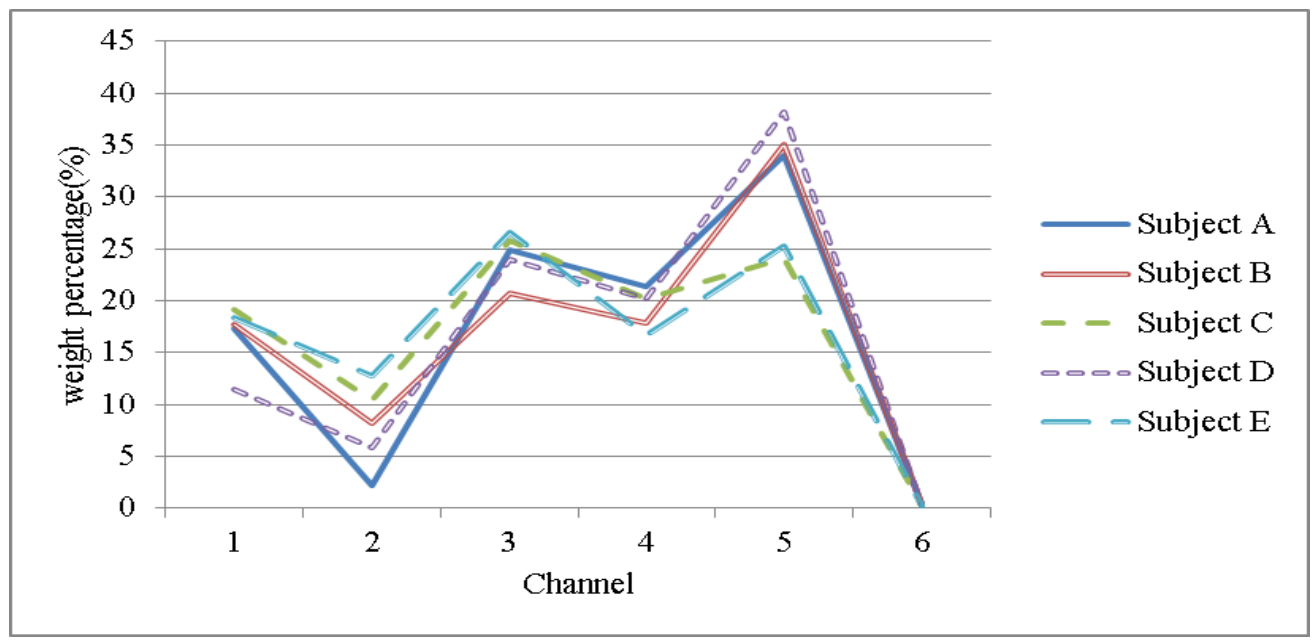

Figure 2. The Weight Efficiency Rates of Subjects

The weight rate of channel 6 is close to 0 , which can be seen as an invalid channel. The reason is that the signal of channel 6 is acquired from the lateral deltoid, the participation level of which in executing different motions is not high. Meanwhile, this muscle is located at the outer shoulder, which makes it difficult to place sensors and avoid interference from cable friction. The weight rates of channel 3, 4 and 5 are relatively high for the positions of these muscles benefit the signal acquisition and they have great differences in executing different motions. Table 1 shows the ordered sequence of the weight percentage.

Table 1. The Order of the Weight Efficiency Rates of Subjects

\begin{tabular}{cl}
\hline Subject & Weight number \\
\hline A & Channel number \\
B & Channel number \\
C & Channel number \\
D & Channel number \\
E & Channel number \\
\hline
\end{tabular}




\subsection{Feature Separability by CSI Value}

The SCI value is used to evaluate the feature separability of different channel combinations. Six channel combinations are obtained by removing the last channel in the weight efficiency rate sequence one by one. Figure 3 shows the CSI values of different channel combinations for five subjects. In the process of channel reduction of each subject, the general trends of feature space separability are the same. For all subjects, the weight of channel 6 is significantly less than other channels, which is the first channel to be reduced. It can be seen from Figure 3 that the separability changes little when the channel 6 is reduced. Therefore, channel 6 is an invalid channel. With further reduction of the channel number, the overall separability of all subjects increases slowly. The reasons are: on the one hand, there may have a great relevance between the reduced channel and one of the remaining channels (the effectiveness of the former is greater than the latter); on the other hand, the reduced channel may have an negative effect on the separability, and a certain increase of the separability for the combination of remaining channels will be observed when this channel is reduced. When the number of channels is reduced to 2 or 3 , the separability of each subject achieves the strongest level.

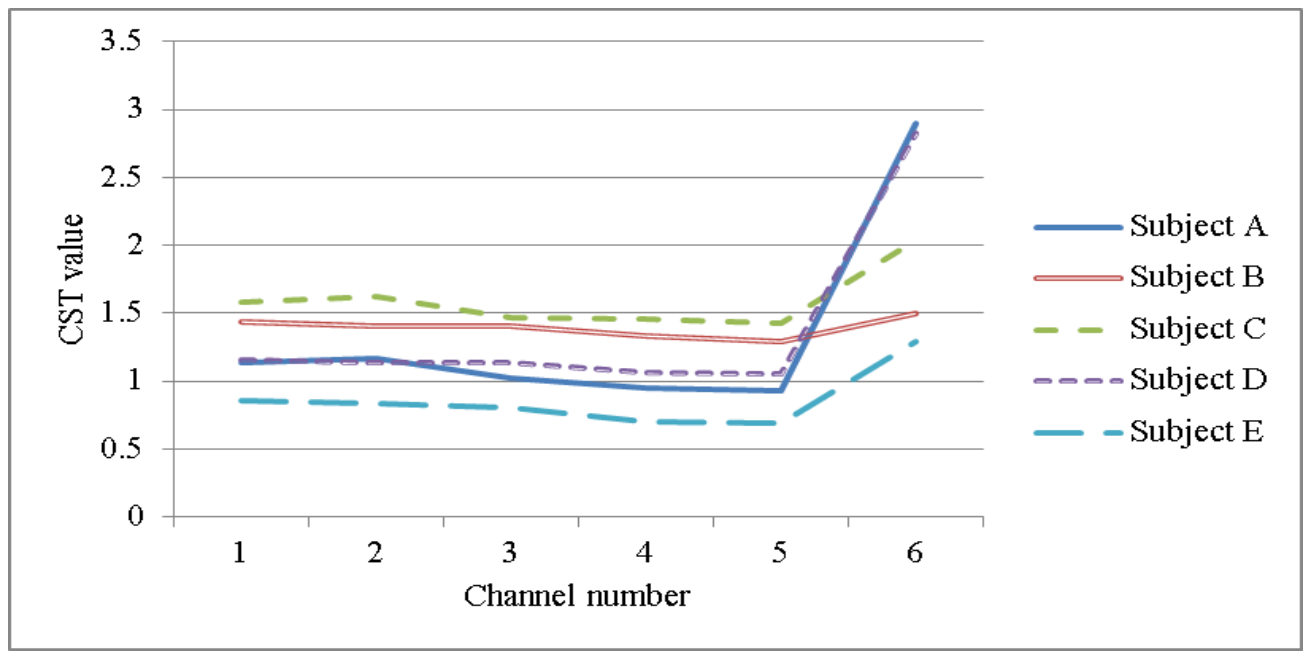

Figure 3. The CSI Value of Different Channel Combinations of Subjects

\subsection{The Results of Channel Reduction}

The purpose of channel reduction is to obtain a higher classification performance with fewer channels. In Section 3.2, when the number of channels is 2 or 3, the separability reaches the highest level. Considering the real-time, accuracy and consistency between the subjects of pattern recognition, the final channel number for combination is considered as 2 . Table 2 shows the reduced channel combination of each subject and the final channel combinations (channel 3 and 5, in different orders for each subject), which guarantees the result of channel reduction consistent among all subjects.

Table 2. The Final Channel Combinations of Subjects After Channel Reduction Expressed by Channel Number

\begin{tabular}{cccccc}
\hline Subject & Subject A & Subject B & Subjcet C & Subject D & Subject E \\
\hline Final Channel & 5,3 & 5,3 & 3,5 & 5,3 & 3,5 \\
\hline
\end{tabular}


Table 3 shows the mean values of correlation between the remaining channels. The correlation coefficients of the remaining channels after channel reduction are less than 0.3 , which are small enough to regard that the two channels are independent from each other without redundancy. After channel reduction, the final feature space is combined by features from channel 3 and channel 5, which contains six dimensions with three features per channel.

\section{Table 3. The Correlation Coefficient of Subjects between Channel 3 and Channel 5}

\begin{tabular}{crrrrr}
\hline Subject & Subject A & Subject B & Subject C & Subject D & Subject E \\
\hline correlation coefficient & 0.2888 & 0.179 & 0.1298 & 0.246 & 0.1861 \\
\hline
\end{tabular}

\section{Discussion}

The purpose of channel reduction is to enhance the real-time and accuracy of motion recognition using as few channel as possible. In this section, the effectiveness of channel reduction is discussed according to classification accuracy, classification speed and application improvement.

\subsection{Classification Accuracy}

The classification accuracy is a very important aspect of the subsequent clinical application [20]. High classification accuracy is the precondition of channel reduction. Figure 4 shows the classification results by SVM before and after channel reduction. For all subjects, the motion classification accuracies have little changes after channel reduction. In other words, channel reduction does not adversely affect the classification accuracy. This result indicates that the number of channels is not the larger the better, which means excessive channels sometimes reduce the overall classification effectiveness. Whereas, the feature space constructed after channel reduction contains enough information about the upper limb motions. The features in the reduced channels contain redundant and ineffective information, which may goes against the overall feature space separability. Therefore, it is feasible and necessary to exclude these channels. The feasibility and effectiveness of the channel reduction are preliminarily proved.

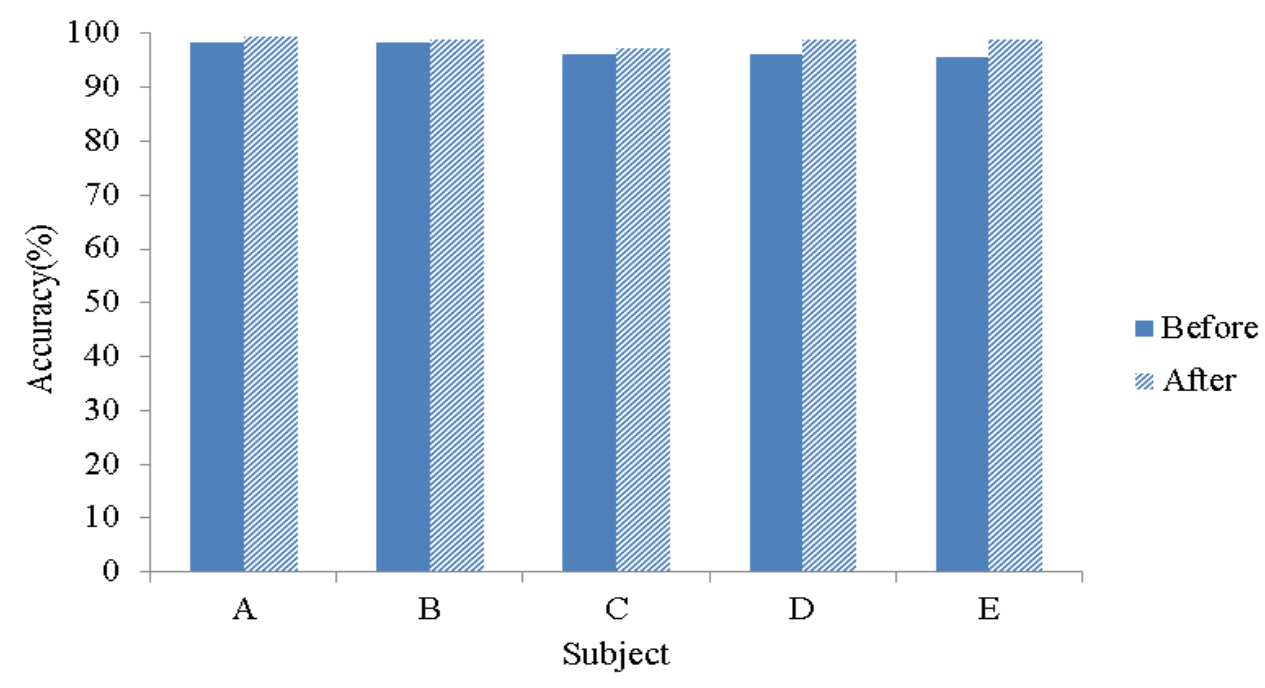
Figure 4. The Impact on Motion Classification Accuracy by Channel
Reduction of Subjects 


\subsection{Classification Speed}

The speed of motion classification is important for real-time applications, which is also one of the main aims of channel reduction [21-23]. The number of channels participated in motion classification has been reduced from 6 to 2 by channel reduction, which inevitably has an impact on the classification speed. It is well known that the pattern recognition mainly consists of three processes, i.e. feature extraction, classifier training and decision-making, which are also the factors affecting the motion classification. In this section, the influence on the classification speed by channel reduction will be evaluated from these three aspects.

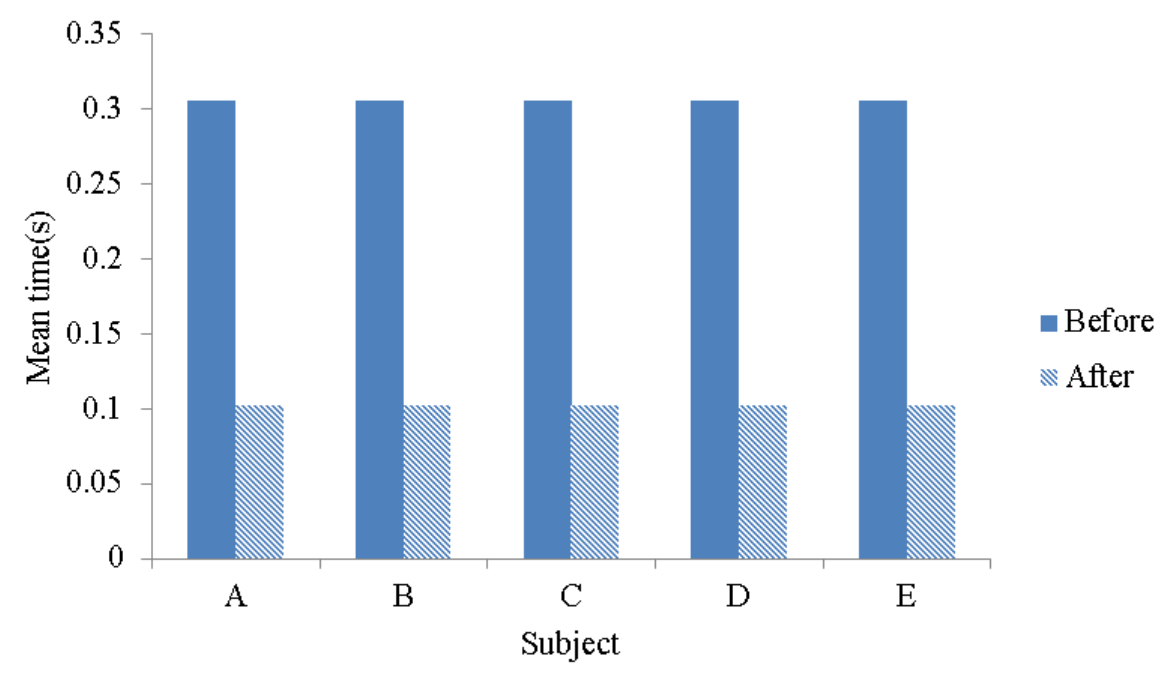

Figure 5. The Impact on Feature Extraction Time from Single Sample by Channel Reduction of Subjects

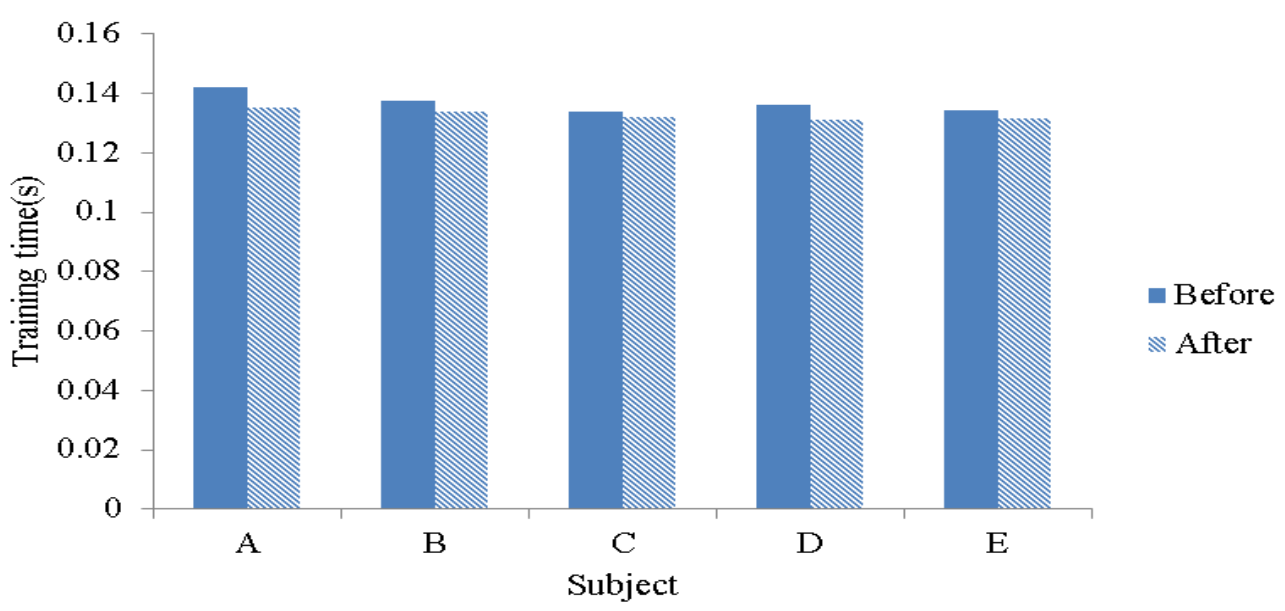

Figure 6. The Influence on Classifier Training Time by Channel Reduction 


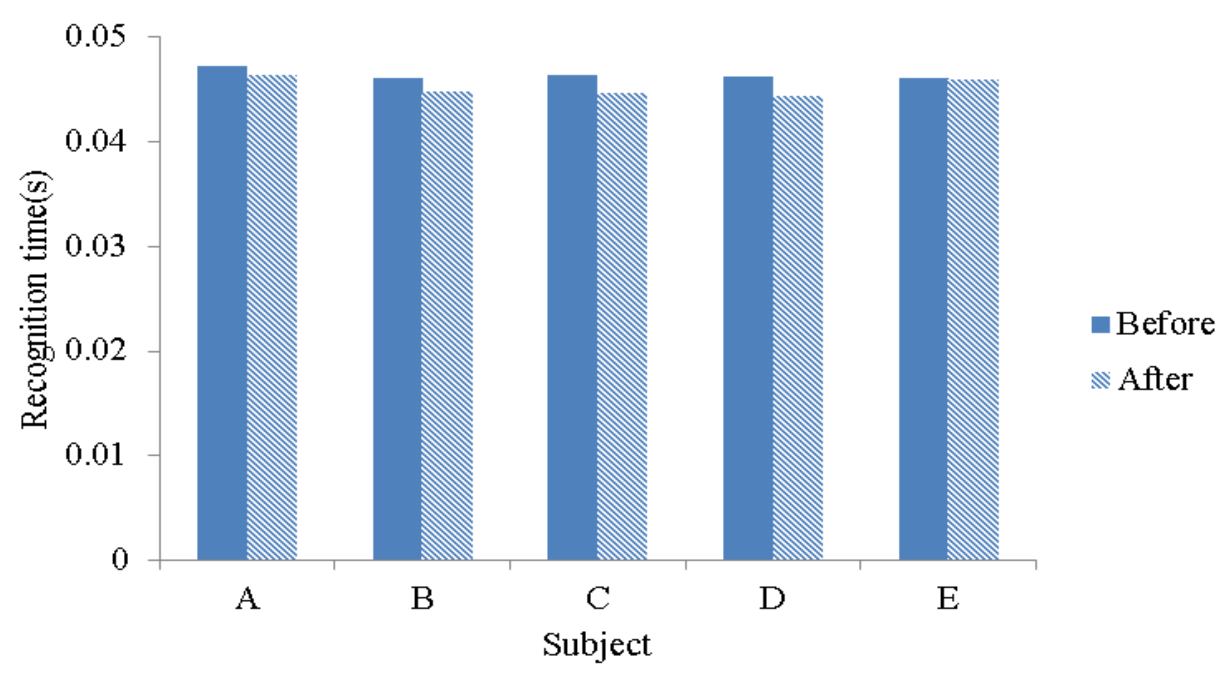

Figure 7. The Impact on Motion Recognition Time by Channel Reduction of Subjects

\subsubsection{Feature Extraction Speed}

Figure 5 shows the average time of the feature extraction for a single sample. Each subject has a consistent performance. After channel reduction, the feature extraction time for a single sample decreases significantly (33\% of that before reduction), which indicates that the extraction speed has been improved. The reason is that the number of channels has been reduced from 6 to 2 after channel reduction. Correspondingly, the feature diminution reduced from 18 to 6 , which means the decreasing range is about $66 \%$. In this case, the number of features needed to calculate is reduced, which reduces the entire computational complexity of feature extraction and increases the speed of feature extraction.

\subsubsection{Classifier Training Speed}

Figure 6 shows the time consumptions of the motion classifier training before and after channel reduction with the training samples. The result indicates that after channel reduction, the training time of motion classifier reduces slightly, which suggests that the classifier training speed is insensitive to feature dimensions of the samples.

\subsubsection{Motion Recognize Speed}

Figure 7 shows the time consumptions of the motion recognition with a trained classifier before and after channel reduction for the testing samples. Similar to the motion classifier training, the time consumptions of motion recognition is reduced slightly after channel reduction.

The above experimental results indicates that the time of overall classification process mainly spent on feature extraction and classifier training, while motion recognition time is relatively less. After channel reduction, as one of the main factors of time consumption, the feature extraction speed increases significantly, although the classifier training speed and the motion recognition speed rise slightly. Meanwhile, the entire motion classification speed has been improved significantly after channel reduction. Therefore, the proposed channel reduction method is valid. By conducting this method in this study, the channel number has been reduced from 6 to 2, which is consistent with the previous studies [24]. Practically, the results help to promote clinical control applications in real-time. 
The channel reduction improves the overall motion classification speed without adversely affecting the classification accuracy. Besides, there are some other effects: the number of channel is reduced after channel reduction, which makes the signal acquisition more convenient. At the same time, it simplifies the structure of the post-rehabilitation training products, which is more conducive to the development of portable applications in clinical rehabilitation and also helps to reduce post-development costs. The method proposed can be extended to other related research areas to realize multivariable choices.

\section{Conclusion}

The classification accuracy and real-time ability are two essential aspects of motion classification. In this article, the signal channel reduction method is proposed and the channel number is reduced significantly. The results show that the overall motion classification speed is improved more without adversely affecting the classification accuracy. The proposed method provides a theoretical basis to channel reduction and a reference for related researches, which also has an important implication on the real-time of sEMG signal based upper limb rehabilitation applications. In addition, the channel reduction not only brings convenience to signal acquisition, but also is conducive to the development of portable clinical rehabilitation products, as well as reducing costs. However, the number and type of test samples is limited in the current research. In the future research, the sample number needs to be expanded, which should cover different ages and genders as well as clinical patients.

\section{Acknowledgments}

Authors will be thankful to the Higher Educational Science and Technology Program of Shandong Province, China (No.J15LB01) and the Natural Science Foundation of Shandong Province, China (ZR2014EEQ029, ZR2015FM021) in carrying out this research for support.

\section{References}

[1] C. La, B. M. Young, C. G. Ramos, V. A. Nair, and V. Prabhakaran, "Characterizing Recovery of the Human Brain following Stroke: Evidence from fMRI Studies", Seeman P, Madras B (eds) Imaging of the Human Brain in Health and Disease, Academic Press/Elsevier, United States, Chapter Twenty, (2014), pp. 485-506.

[2] B. Balaban, F. Tok, F. Yavuz, E. Yaşar and R. Alaca, "Early rehabilitation outcome in patients with middle cerebral artery stroke", Neuroscience letters, vol. 498, no. 3, (2011), pp. 204-207.

[3] P. Langhorne, J. Bernhardt and G. Kwakkel, "Stroke rehabilitation", The Lancet, vol. 377, no. 9778, (2011), pp. 1693-1702.

[4] J. L. Patton, M. E. Stoykov, M. Kovic and F. A, "Mussa-Ivaldi. Evaluation of robotic training forces that either enhance or reduce error in chronic hemiparetic stroke survivors", Exp Brain Res, vol. 168, no. 3, (2006), pp. 368-383.

[5] C. D. Takahashi, L. Der-Yeghiaian, V. Le, R. R. Motiwala and S. C. Cramer, "Robot-based hand motor therapy after stroke", Brain, vol. 131, no. 2, (2008), pp. 425-437.

[6] L. Zollo, L. Rossini, M. Bravi, G. Magrone, S. Sterzi and E. Guglielmelli, "Quantitative evaluation of upper-limb motor control in robot-aided rehabilitation", Medical \& Biological Engineering \& Computing, vol. 49, no. 10, (2011), pp. 1131-1144.

[7] W. S. Harwin, A. Murgia and E. K. Stokes, "Assessing the effectiveness of robot facilitated neurorehabilitation for relearning motor skills following a stroke", Medical \& Biological Engineering \& Computing, vol. 49, no. 10, (2011), pp. 1093-1102.

[8] E. N. Kamavuako, E. J. Scheme and K. B. Englehart, "Combined surface and intramuscular EMG for improved real-time myoelectric control performance", Biomedical Signal Processing and Control, vol. 10, no. 3, (2014), pp. 102-107.

[9] M. B. I. Raez, M. S. Hussain and F. M. Yasin, "Techniques of EMG signal analysis: detection, processing, classification and applications", Biological procedures online, vol. 8, no. 1, (2006), pp. 1135 . 
[10] Y. Li, X. Chen, X. Zhang and P. Zhou, "Several practical issues toward implementing myoelectric pattern recognition for stroke rehabilitation", Medical Engineering \& Physics, vol. 36, no. 6, (2014), pp. 754-760.

[11] Khushaba RN, Kodagoda S, Takruri M and Dissanayake G, "Toward improved control of prosthetic fingers using surface electromyogram (EMG) signals”, Expert Syst Appl, vol. 39, no. 12, (2012), pp. 10731-10738.

[12] Smith A and Brown EE, "Myoelectric control techniques for a rehabilitation robot", Applied Bionics and Biomechanics, vol. 8, no. 1, (2011), pp. 21-37.

[13] Shenoy, P., Miller, K.J., Crawford, B., and Rao, R.P., "Online electromyographic control of a robotic prosthesis", IEEE Transactions on Biomedical Engineering, vol. 55, no. 3, (2008), pp. 1128-1135.

[14] Shuling. Bai, "System anatomy", People's Medical Publishing House, Beijing, (2001).

[15] Andrews A, Morin E and McLean L, "Optimal Electrode Configurations for Finger Movement Classification using EMG", Annual International Conference of the IEEE in Engineering in Medicine and Biology Society, Minneapolis, USA, (2009)September 3-6.

[16] Ryait HS, Arora AS and Agarwal R, "SEMG signal analysis at acupressure points for elbow movement", Journal of Electromyography and Kinesiology, vol. 21, no. 5, (2011), pp. 868-876.

[17] Kira K and Rendell L, "Feature selection problem: traditional methods and a new algorithm", Proceedings Tenth National Conference on Artificial Intelligence - AAAI-92, San Jose, Ca, USA, (1992) July 12-16.

[18] Yanzhao. Chen, Xiangli. Cheng, Xiaohua. Fan and Yuwei Zhang, "Bispectrum-based sEMG multidomain joint feature extraction for upper limb motion classification”, Proc IMechE Part C: J Mechanical Engineering Science, vol. 230, no. 2, (2016), pp. 1989-1996.

[19] I. Kononenko, "Estimating attributes. Analysis and extensions of RELIEF", Proceedings of the European Conference on Machine Learning, Lecture Notes in Artificial Intelligence, Catania, Italy, (1994).

[20] M. A. Oskoei and H. H. Sheng, "Myoelectric control systems-A survey", Biomedical Signal Processing and Control, vol. 2, no. 4, (2007), pp. 275-294.

[21] M. Zecca, S. Micera, M. C. Carrozza and P. Dario, "Control of multifunctional prosthetic hands by processing the electromyographic signal", Critical Reviews ${ }^{\mathrm{TM}}$ in Biomedical Engineering, vol. 30, no. 46, (2002), pp. 459-485.

[22] Z. O. Khokhar, Z. G. Xiao and C. Menon, "Surface EMG pattern recognition for real-time control of a wrist exoskeleton", Biomedical engineering online, vol. 9, no. 1, (2010), pp. 41.

[23] T. R. Farrell and R. F. Weir, "The Optimal Controller Delay for Myoelectric Prostheses", IEEE Trans Neural Syst Rehabil Eng, vol. 15, no. 1 (2007), pp. 111-118.

[24] E. L. Glassman and J. V. Guttag, "Reducing the number of channels for an ambulatory patient-specific EEG-based epileptic seizure detector by applying recursive feature elimination", 28th Annual International Conference of the IEEE in Engineering in Medicine and Biology Society, New Jersey, United States, (2006).
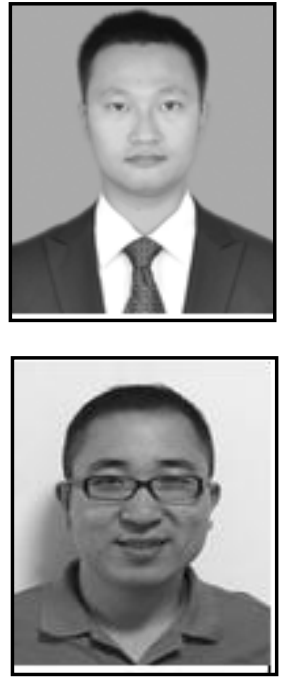

\section{Authors}

Yanzhao Chen, received the Dr. degree from school of mechanical engineering, Shandong University, China, in 2015. $\mathrm{He}$ is currently working in school of mechanical and automotive engineering, Qilu University of Technology. His research interests include signal processing and computer graphics.

Yu-Wei Zhang, associate professor and Master instructor in school of mechanical and automotive engineering, Qilu University of Technology. His research interests include computer graphics and digital geometry processing. 